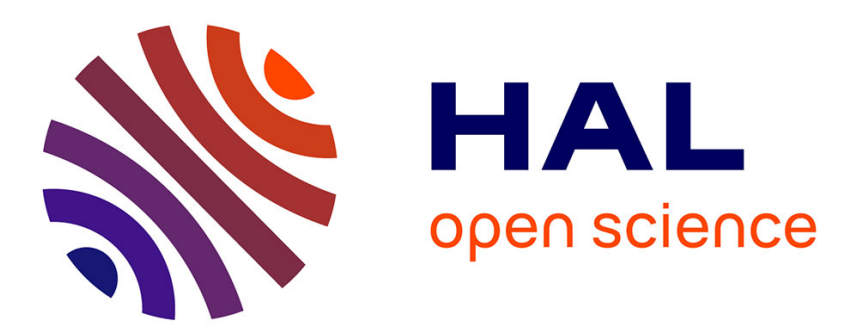

\title{
Tests and Confidence Intervals for a Class of Scientometric, Technological and Economic Specialisation Ratios
}

\author{
Torben Schubert, Hariolf Grupp
}

\section{- To cite this version:}

Torben Schubert, Hariolf Grupp. Tests and Confidence Intervals for a Class of Scientometric, Technological and Economic Specialisation Ratios. Applied Economics, 2009, 43 (8), pp.941. 10.1080/00036840802600160 . hal-00582254

\section{HAL Id: hal-00582254 \\ https://hal.science/hal-00582254}

Submitted on 1 Apr 2011

HAL is a multi-disciplinary open access archive for the deposit and dissemination of scientific research documents, whether they are published or not. The documents may come from teaching and research institutions in France or abroad, or from public or private research centers.
L'archive ouverte pluridisciplinaire HAL, est destinée au dépôt et à la diffusion de documents scientifiques de niveau recherche, publiés ou non, émanant des établissements d'enseignement et de recherche français ou étrangers, des laboratoires publics ou privés. 


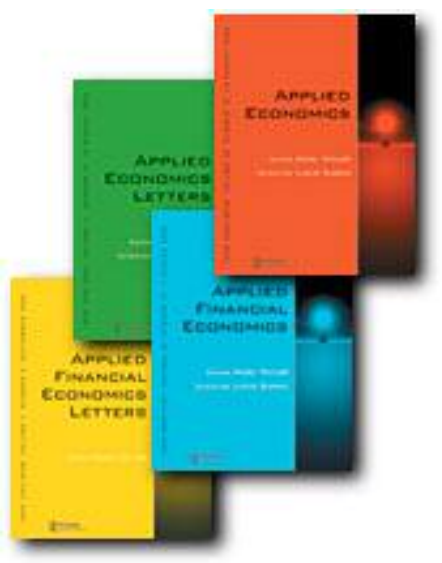

Tests and Confidence Intervals for a Class of Scientometric, Technological and Economic Specialisation Ratios

\begin{tabular}{|c|c|}
\hline Journal: & Applied Economics \\
\hline Manuscript ID: & APE-07-0472.R1 \\
\hline Journal Selection: & Applied Economics \\
\hline $\begin{array}{r}\text { Date Submitted by the } \\
\text { Author: }\end{array}$ & 03-Jul-2008 \\
\hline \multirow[t]{2}{*}{ Complete List of Authors: } & $\begin{array}{l}\text { Schubert, Torben; Fraunhofer Institute for Systems and Innovation } \\
\text { Research } \\
\text { Grupp, Hariolf; University of Karlsruhe, Institute for Economic Policy } \\
\text { Research }\end{array}$ \\
\hline & $\begin{array}{l}\text { C12 - Hypothesis Testing < C1 - Econometric and Statistical } \\
\text { Methods: General < C - Mathematical and Quantitative Methods, } \\
\text { O30 - General < O3 - Technological Change|Research and } \\
\text { Development < O - Economic Development, Technological Change, } \\
\text { and Growth, C13 - Estimation < C1 - Econometric and Statistical } \\
\text { Methods: General < C - Mathematical and Quantitative Methods }\end{array}$ \\
\hline Keywords: & specialisation, indicators, statistical inference, RPA, RLA \\
\hline
\end{tabular}

\section{๑) ScholarONE" \\ Manuscript Central}




\title{
Tests and Confidence Intervals for a Class of Scientometric, Technological and Economic Specialisation Ratios
}

\author{
Torben Schubert12 and Hariolf Grupp 3
}

\begin{abstract}
In economic, scientometric, and innovation research, often so-called specialisation indices are used. These indices measure comparative strengths or weaknesses as well as specialisation profiles of the observation units with respect to certain criteria, such as patenting and publication or trade activities. They allow question like: Is Germany specialised in the export of motor vehicles? Or is the UK specialised in biotech patents? Unfortunately, little is known about their statistical properties, which makes valid inferencing difficult. In this article we prove asymptotic normality for a certain class of scientometric, technological, and some economic, though non-monetary, specialisation indices. We provide asymptotic confidence intervals and demonstrate in an example how to obtain statistically sound results. We will also address the problem of normalisation of these indicators. All procedures proposed are provided in an add-on package for R statistical environment.
\end{abstract}

1 Fraunhofer Institute for Systems and Innovation Research (ISI), Karlsruhe, Germany, Institute for Economic Policy Research (IWW), Karlsruhe University, Germany

2 Corresponding author, email: torben.schubert@isi.fraunhofer.de

3 Fraunhofer Institute for Systems and Innovation Research (ISI), Karlsruhe, Germany, Institute for Economic Policy Research (IWW), Karlsruhe University, Germany 


\section{Introduction}

Information about countries' scientific, technological, or economic performance is often required for various reasons. The specific strengths and weaknesses are commonly assessed by indicators. Thus, for example, a country's patent count will be interpreted as a measure of its technological capacity (Blind and Jungmittag, 2005, Jang et al., 2008). Other studies focus on scientific publications to assess a country's scientific performance (Garcia-Castrillo et al., 2002). Apart from using simple counts as performance measures, also specialisation ratios, describing a country's profile (e.g. specialisation in high-tech or low-tech patents; specialisation in natural science or social science publications; export and import profiles), are widely applied (e.g. Jensen, 2002, De Benedictis et al., 2008)

Originally specialisation ratios were introduced in the context of the analyis of trade flows. Among the early indicators are the Revealed Export Advantage (RXA) and the Revealed Import Advantage (RMA) (Keesing, 1965). Another example is the the Revealed Comparative Advantage (RCA) (see Balassa, 1965, 1977, Wolter, 1977, De Benedictis and Tamberi, 2001, for a discussion). Other indicators that describe trade flows emerge from the phenomenon of intraindustry trade (IIT). The earliest indicator measuring IIT is the Grubel-Lloyd Index (GLI) (Grubel and Lloyd, 1971, 1975). Many more IIT indicators have been developed in this branch of research (an overview can be found in Brülhart (1994)). ${ }^{4}$ Later on, in technometric and scientometric research similar indicators originated from the methodogical ideas of the RMA, the RXA, and the RCA, such as the Revealed Patent Advantage (RPA) and the Revealed Literature Advantage (RLA) (Grupp, 1998, pp. 157 - 158, 216 - 219). Indicators also emerged in economic analysis, including the Revealed Trade Mark Advantage (RTMA) and the Revealed Norms and Standards

4 Note for now that the GLI and, related to it, other IIT indicators are not really specialisation ratios, as they are discussed in this paper. This will become clear later on. 
Specialisation (RNSS). Additionally, the TRIS database containing national regulations of EUmembers has been used to calculate the Revealed Regulation Specialisation (RRS). 5 All these indicators have a neutral value which indicates neither above nor below average specialisation. Values below the neutral value are a sign of under-specialisation, whereas values above the neutral indicate over-specialisation.

Due to their easy construction and interpretability they have awakened growing interest in various fields of research, as they provide information about the relative strengths of certain units in measuring specialisation without reference to size. Nonetheless, in a statistical sense, specialisation ratios are complex sample functions, for which the stochastic properties are largely unknown. While there is a formula to calculate some form of "parametric imprecision" (Engelsman and van Raan, 1990), the inability to determine their statistical distribution makes interpretation a cumbersome endeavour, often subjected to intuition or rules of thumb. In the following we will provide the asymptotic distribution for a specific class of specialisation ratios. Commonly, though not exclusively, these specialisation ratios will be technometric or scientometric.

The rest of the paper is organised as follows. Section 2 presents the most common specialisation ratios, to provide an intuition for the interpretation of these indicators. In view of their importance we will also describe the trade-related monetary specialisation ratios, although they are not covered by the statistical analysis of this paper. Section 3 summarises the main statistical results. The derivations may be found in the appendix. Section 4 presents an empirical example and some considerations of robustness with respect to classification decisions. Section 5 concludes.

5 http://ec.europa.eu/enterprise/tris/. 


\section{Common Specialisation Ratios}

In this section, we discuss the most common indicators and their meaning in order to provide the reader with a feeling for this type of indicators. We also discuss which indicators meet the conditions needed for the statistical analysis and which do not. Before we do so, we introduce a technical definition and the requirements formally:

Technical Definition: A specialisation ratio is a measure which divides a ratio describing some feature of unit under consideration by an identically constructed ratio corresponding to a reference unit.

Statistical Conditions: (a) The quantities concerned are measured on an absolute scale.6 (b) The specialisation ratio can be interpreted as a fraction of fractions. ${ }^{7}$

To begin with, any indicator adhering to the technical definition and the statistical conditions is a specialisation ratio that falls in the class of indicators for which we are able to determine the asymptotic distribution by the methodology used in this paper.

We start our review of the most common specialisation indicators with those that either fail the technical definition or the statistical conditions (or both). Among the earliest specialisation ratios is the Revealed Comparative Advantage (RCA) - a measure for comparative advantage - which is given by 8

$$
R C A_{i k}=\left(E_{i k} / I_{i k}\right) /\left(\sum_{h} E_{h k} / \sum_{h} I_{h k}\right)
$$

6 Note that quantities that can be measured on an absolute scale have a natural unit. In our context this will imply that the quantities can be counted (for example, patents).

7 The technical definition only requires it to be a ratio of ratios.

8 Check that the technical definition applies. 
where $E$ and $I$ denote exports and imports in a given product group and a given country. The RCA compares the export to import ratio of some country $i$ for product group $k$ to that of the reference group of countries. A value greater than 1 is indicative of a comparative advantage the country $i$ has with respect to product group $k$, while a value below 1 is a sign of a comparative disadvantage. Concerning requirements (a) and (b), neither the numerator nor the denominator is assured to be between zero and one, so that none is a fraction. Further, the quantities of the RCA fail the condition of having a natural unit. This is easy to see, by considering the following idea: a count measure could be derived by taking each euro as an individual element. Unfortunately, though unit stepped, this is not unique since the imports or exports could also be measured in yen or dollars. By changing the scale we would also change the number of counts.

The Revealed Export Advantage (RXA) - a measure for export specialisation - and the Revealed Import Advantage (RMA) - a measure for import specialisation - are defined as 9

$$
R X A_{i k}=\left(E_{i k} / \sum_{j} E_{i j}\right) /\left(\sum_{h} E_{h k} / \sum_{h} \sum_{j} E_{h j}\right)
$$

and

$$
R M A_{i k}=\left(I_{i k} / \sum_{j} I_{i j}\right) /\left(\sum_{h} I_{h k} / \sum_{h} \sum_{j} I_{h j}\right)
$$

For instance, if the RMA is greater than unity, then country $i$ imports more of product group $k$ in comparison to own total imports than does the reference group of countries compared to its total imports. Country $i$ imports more of product group $k$, relatively, than does the rest of the world. Clearly, the fractions condition is met. Unfortunately, both the RMA and the RXA fail the natural unit condition for the same reason as the RCA does.

9 In this definition $\sum_{j} E_{i j}$ denotes total exports of country $i$. 
Despite these more traditional trade indicators, many advances have been made in measuring intra-industry trade (ITT). The earliest indicator of this type is the Grubel-Lloyd-Index (GLI), which we will shortly discuss here. The GLI is defined by following formula:

$$
G L I_{i k}=\frac{\sum_{j}\left(E_{i j}+I_{i j}\right)-\left|\sum_{j} E_{i j}-\sum_{j} I_{i j}\right|}{\sum_{j}\left(E_{i j}+I_{i j}\right)}
$$

where the notation is the same as above. The index takes a value of unity whenever all trade is intra-industrial and zero if all trade is inter-industrial. In fact, the GLI is not a specialisation ratio, as this was defined previously (see the technical definition). The reason for mentioning it here is the problem of aggregation bias, which has a parallel in what will be discussed later in Section 4.2. For the sake of completeness, it shall be noted that neither condition (a) nor condition (b) applies.

We now turn to indicators that meet the technical definition and the statistical requirements. The Revealed Patent Advantage (RPA) - a measure for technological specialisation proxied by patents - and the Revealed Literature Advantage (RLA) - a measure of science specialisation proxied by scientific publications - are defined very similarly to the RXA and the RMA. Take the RLA, for instance:

$$
R L A_{i k}=\left(P_{i k} / \sum_{j} P_{i j}\right) /\left(\sum_{h} P_{h k} / \sum_{h} \sum_{j} P_{h j}\right)
$$

where $P$ denotes publications. A value of above 1 means that the fraction of publication in discipline $k$ (e.g. biotechnology) of country $i$ (say, the $\mathrm{UK}$ ) is greater than the respective fraction of the reference region (e.g. the world). To put it in simple terms: the UK gives, in this hypothetical example, much greater relative emphasis to biotechnology compared to the rest of the world. Thus the fraction condition is met, as with the RMA and the RXA. Also, the number of publications is a natural integer stepped unit. The same holds true for the RPA. Other measures with 
identical properties are specialisation ratios based on trade marks (RTMA), norms and standards (RNSS), and technical regulation (RRS), which are certainly economic rather than scientometric or technological indicators.

In summary, the procedures discussed subsequently work well, whenever quantity describing the feature can be (uniquely) counted and classified. This applies for example for the RPA, the RLA, the RTMA, the RNSS, and the RRS, but not for he RXA, the RMA or the RCA and not for any kind of monetary indicator such as the GLI. So the main restriction in an economic sense is that the indicators must be non-monetary.

Another issue concerns interpretability. The fact that all these indicators range between zero and infinity with neutral value at 1 makes interpretation a cumbersome endeavour. Symmetric and normalised indices are easier to understand. Therefore, often the symmetrifying second order Moebius transformation in the form $\left(r^{2}-1\right) /\left(r^{2}+1\right)$, where $r$ is the respective specialisation index, is embarked upon (Grupp, 1994, pp. 187 onwards). The transformed index then ranges from -1 to +1 with a neutral value ("no specialisation") at 0 . However, there is a large class of similar Moebius transformations, 10 and to our knowledge, there is no mathematical solution to determine the optimal transformation so far. ${ }^{11}$ In the following analysis we adopt the second order version, for simplicity's sake. In any case, this is not a great problem in our context because our results hold for any differentiable transformation, which is true for any transformation common to the literature.

10 Note that $\left(r^{k}-1\right) /\left(r^{k}+1\right)$ for any $k>0$ has the same properties. Apart from the Moebius transformations there are others with identical normalisation properties.

11 Also confirmed in a private communication from Dr. Markus von Ins, CEST, Switzerland, on September 11, 2006. 


\section{Asymptotic Results}

In this section we briefly present the main theorems derived in this paper. The proofs are given in the Appendix. All of the procedures are implemented in the R-package SRinference (current version: 1.1) freely available under http://www.isi.fhg.de/p/mitarbeiter/tos.html, which does not require much statistical knowledge to use it. Additionally, a user guide is provided, which introduces the basic features of the package to the user. Therefore, for the application-oriented reader these derivations may be skipped as well as the theorems in Section 3. Instead, Section 4 gives an empirical example. This should help to make application and interpretation more accessible.

Let us first introduce some definitions. We will refer to 'unit' when we mean the unit having certain attributes. We will denote as 'objects' the attributes which may take certain values. For example, when we talk about Germany's strength in publishing reviewed articles in the field of natural sciences, Germany is the unit while a given reviewed article is the object (which either belongs to natural sciences or not).

The theorems only present the (asymptotic) distributions of the statistics under consideration. In any case, this is enough for our purpose because once their distribution is known to be normal, it is straightforward to define valid tests and confidence intervals, following the spirit of usual ttests or t-based intervals.

We define the following variates:

$X_{i j} \quad$ A random variable, which is 1 , whenever object $i$ for unit $j$ belongs to the field under consideration and 0 otherwise 12

12 We use either $k$ or $v$ throughout the text to denote the subjects under consideration. Also note that by defining an individual object we make use of the 'absolute scale' condition. 
$\sum_{i=1}^{n_{j}} X_{i j}$

A random variable which counts the number of all objects for unit $j$ which belong to the field under consideration

When different times are concerned, the objects and their number will be denoted by $X_{i j}^{k}$ and $n_{j}^{k}$ where the superscript indicates the time period.

During applied work it is often necessary to determine if a country is specialised e.g. in patenting activity in the chemical industry field. However, for values of the RPA close to zero, it is hard to maintain that the observed deviation is not just coincidental. Therefore a one-sample test is useful to determine whether the observed effect is not just noise. The result of Theorem 1 allows such inference, including, of course, the construction of confidence intervals.

Theorem 1 (One Sample Inference): If i) $n_{j}$ is non-random for all $j$, ii) $X_{i j}$ is independent of $X_{h l}$ whenever both variates denote different objects, iii) when $n$ increases, also each $n_{j}$ grows, such that $n / n_{j} \rightarrow$ const, iv) the function used to transform the linear indicator is differentiable around the estimated value, v) (a) the objects have a natural unit and (b) the indicator has a fraction of fractions meaning, then the transformed specialisation ratio is asymptotically normal and $\sqrt{n}$-consistent with a variance that can be consistently estimated from the data.

Especially assumption ii) is very strong. It essentially assumes away any kind of economic reasons for dependence. Taking the example of patenting activity, it means that regional clustering of industrial (and therefore innovative) activity is neglected, despite the fact that its existence is widely accepted in the literature (Krugman, 1991). There are a multitude of reasons for that, including spill-overs, endowment with natural or human resources, demand and supply side externalities resulting from transportation costs, pathdependence, etc. Yet the independence assumption is unavoidable from a statistical point of view (see Appendix). The reader is encouraged to 
decide if in a specific application this assumption seems justified or if it is too strong. This will also depend on how strong regional interdependencies are believed to be.

Very often we would like to test whether two specialisation ratios are significantly different or not. For example, we might like to know if Italy's specialisation in astrophysical publications is significantly different from that of Portugal. This is also possible as indicated in Theorem 2.

Theorem 2 (Two Sample Inference): Under the conditions of Theorem 1, the difference of the transformed specialisation ratio is asymptotically normal and $\sqrt{n}$-consistent with a variance that can be consistently estimated from the data.

Sometimes changing structures in time are relevant. We may, for example, want to know if the patent specialisation of the UK concerning biotechnology has significantly changed over time. Theorem 3 gives conditions under which we can test for this.

Theorem 3 (Time Change Inference): If i) $n_{j}^{k}$ is non-random for all $j$ and $k \in\{s, t\}$, ii) $X_{i j}^{k}$ is independent of $X_{h l}^{v}$ whenever both variates denote different objects, iii) when $n^{t}$ increases, also each $n_{j}^{t}$ and $n_{j}^{s}$ grows, such that $n^{t} / n_{j}^{s} \rightarrow$ const and $n^{t} / n_{j}^{t} \rightarrow$ const, iv) the function used to transform the linear indicator is differentiable around the estimated value, v) (a) the objects have a natural unit and (b) the indicator has a fraction of fractions meaning, then the difference of the transformed specialisation ratios in time is asymptotically normal and $\sqrt{n}$-consistent with a variance that can be consistently estimated from the data.

In essence, Theorems 1-3 define ways to construct confidence intervals and tests for the statistics discussed (let these be denoted as $T_{n}$ ) by approximating the finite sample distribution with its asymptotic counterpart. However, simulation studies have shown that this approximation may be quite imprecise if the sample size is small. We therefore provide a bootstrap procedure which has 
better rates of convergence (Hall, 1992, Horrowitz, 2001). In essence, this will make inference much more precise in small samples. The consistency results are summarised in Theorem 4.

Theorem 4 (Bootstrap Inference): Under the respective conditions of Theorems 1-3 the resampling bootstrap distribution of any of the statistics $T_{n}$ is a consistent estimate of the true distribution.

From a practical point of view, in small samples we recommend the use of the estimator described in Theorem 4 (function SRboottest in the R-package). However, with large enough samples ( $n>200$ and $n_{j}>15$ for all $j$ ), the analytical tests and confidence intervals from Theorems 1-3 give a reasonably good approximation (function SRtest in the R-package). Under these conditions time savings should outweigh the higher precision of the bootstrap method. 


\section{An Empirical Example and the Problem of Classification}

In this section we present an empirical application and discuss the robustness of the procedure with respect to the definition of the classes.

\subsection{A Short Empirical Example}

To save space, we present only the case of one sample inference. We use the asymptotic test from Theorem 1. In any case, the other procedures work in very much the same manner.

For the empirical example we use patent data on 11 countries gathered for a study of the catchingup process of several of Germany's world market competitors in the context of the Technological Performance Report 2006 prepared for the German Ministry of Education and Research.

One task of this study was to describe the technological profile of these catching-up countries by their patenting profile. 13 The results for the profile of patenting activity are depicted in Figure 1.

To do this, specialisation ratios as described above were used along with the second order Moebius transformation. The latter bounds the specialisation ratio to the interval -1 and 1 . A zero indicates that the country average is identical with the reference group average (often the world average) while positive values indicate above average und negative below average specialisation. 
Figure 1: Patent Profile for 11 catching-up countries in 2003 (including 95\%-confidence intervals)

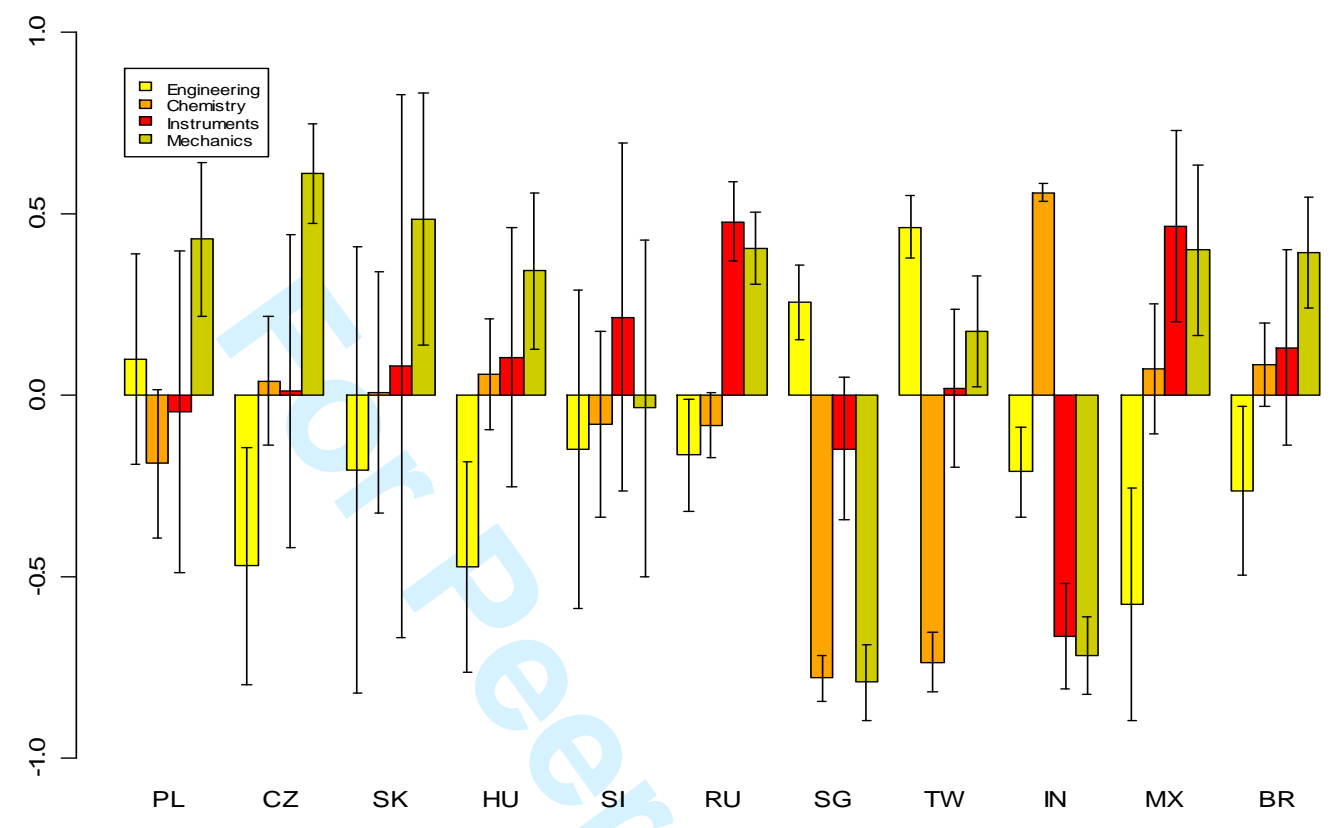

The main results can be summarised as follows. The eastern European countries (Poland, Czech Republic, Slovakia, Hungary, and Russia) have somewhat similar profiles, putting high weight on mechanics. This means that they are still, to some degree, in the production tradition of the Warsaw Pact. Slightly different profiles are apparent for Slovenia, Slovakia, Hungary, and Russia which are specialised in instruments. However, we also see that only the Russian specialisation in instruments is statistically significant, which indicates that the results for the other three countries are not overwhelmingly reliable. Though not applying to Slovenia, where none of the estimated specialisation ratios is statistically significant from zero, the similar profiles across the Eastern European countries are largely real effects. The non-significance in the Slovenian case might have two reasons. First, it does not have a very sharp profile. So indeed, Slovenia might follow a rather 
'generalist' patenting strategy. Second, Slovenia's patent counts are very low. Therefore, the precision of the specialisation ratios is limited. The bands for India, on the contrary, are very narrow, because the total number of India's patents is relatively large (1202).

Analogous to the eastern European cluster there is definitely a South American cluster of Mexico and Brazil, which also share similar profiles. To some extent, Singapore and Taiwan also have similarities.

So the main conclusion of this analysis could be that in fact regional clusters of technological specialisation do exist, that are shaped by regional, technological or cultural proximity and possibly by comparable factor and human capital endowments.

However, knowledge about statistical confidence is still absolutely necessary, in order to separate real effects from purely random ones. The same applies to time change inference. This can help to detect significant changes in country profiles earlier or prevent researchers from interpreting noise as relevant changes.

\subsection{Influence of the Classification}

Motivated by the well-known aggregation bias inherent to the construction of the GLI, 14 it is worthwhile asking what is likely to happen, if the classes of the previous example (engineering, chemistry, instruments, and mechanics) were disaggregated. Intuitively, it can be expected that the significance of specialisation is likely to decrease, because estimations will become less precise. If this were true, then disaggregating patent classes would have a very similar effect to the

14 Aggregation bias in this context means that IIT is overestimated and vanishes after disaggregation The reason is the 'opposite sign effect'. (Gray, 1979, Greenaway, 1983, Tharakan, 1984, Balassa, 1987, Doroodian et al., 1999, Bahmani-Oskooee and Harvey, 2006). 
aggregation bias, though for a very different reason. This question is of special relevance, because classification is largely arbitrary.

Referring to the empirical example of the previous subsection, the chosen level with only four classes is rather broad. Since the International Patent Classification (version 7) contains about 85,000 classes, we could have relied on a much more detailed analysis.

We will demonstrate that the asymptotic variance will increase with the number of the classes considered, implying a decrease of precision and significance. In any case, finding an analytical solution in the general case seems very hard. Therefore we consider only a special case in which, after still tedious calculations, an easy closed form expression pertains.

Taking the test of Theorem 1 along with the second order Moebius transformation, we assume that for a given class, no subject is over- or under-specialised and that each subject holds an identical number of objects. Further, we assume that all classes have the same probability that an object belongs to them both before and after disaggregation.

These assumptions result in the following simplifications:

$\pi_{1}=\pi_{2}=\ldots=\pi_{J}=\pi=1 / K$

$n_{1} / n=n_{2} / n=\ldots=n_{J} / n=1 / J$

where $K$ and $J$ are the number of classes and the number of subjects. Taking the expression for the asymptotic variance of test in Theorem 115 , we see that

$$
\sigma_{I}^{2}=n \sum_{j=1}^{J}\left(\frac{d I}{d r} \frac{\partial r}{\partial \pi_{j}}\right)^{2} \frac{\pi_{j}\left(1-\pi_{j}\right)}{n_{j}}
$$

15 Equation (10) of the Appendix. 


$$
\begin{aligned}
& =\left(\frac{d I}{d r}\right)^{2} \frac{K-1}{K^{2}} J \sum_{j=1}^{J}\left(\frac{\partial r}{\partial \pi_{j}}\right)^{2} \\
& =\left(\frac{d I}{d r}\right)^{2} \frac{K-1}{K^{2}} J\left[\left(\frac{\pi}{\pi^{2}} \frac{1}{J}\right)^{2}(J-1)+\left(\left(\frac{\pi}{\pi^{2}} \frac{1}{J}(J-1)\right)^{2}\right)\right] \\
& =\left(\frac{4 r}{\left(r^{2}+1\right)^{2}}\right)^{2}(K-1)(J-1) \\
& =(K-1)(J-1)
\end{aligned}
$$

where the last line follows from the assumption of no specialisation, i.e. $r=1$. This indicates that the asymptotic standard error increases by the order $1 / 2$ in the number of classes. Therefore, disaggregating too much will increase the asymptotic variance and for any given sample size will result in a drop in significance.

However, unlike the aggregation bias known from the GLI, this is not a measurement bias, but purely a statistical problem of finite samples, which vanishes asymptotically for any finite number of classes.

Still, it should be stressed that the definition of classes should follow an economically sensible understanding and should result in a manageable number of classes, because otherwise the precision of the specialisation ratios is lost. 


\section{Conclusion}

Specialisation ratios are in wide use concerning science, technology and trade assessment. Up to now, little was known about their statistical reliability. In order to progress in this field, we proved asymptotical normality for a certain type of non-monetary specialisation ratios. We used this knowledge to provide asymptotic confidence intervals and tests for the one and two-sample problem and the two-sample time change problem, whose critical values converge at usual rates, obtained from first order asymptotics. We showed in an empirical example how this knowledge can be applied to obtain statistically sound results. As specialisation ratios are a widely accepted und frequently used tool in economics, scientometrics, and technometrics, we believe that these new inferencing techniques are extremely relevant to any user of such indicators. In order to guarantee fast dissemination, we implemented each method proposed in an add-on package for $\mathrm{R}$

\section{Acknowledgements}

The research underlying this paper was partially supported by the German Research Foundation (Deutsche Forschungsgemeinschaft, DFG) in a project on "performance indicators for research groups" (SCHM 1719/1-2) which is part of a larger research group on "international competitiveness and innovation capacity of universities and research institutions - new forms of governance" (FOR 517). We would also like to thank two anonymous referees who with their comments helped significantly to improve the quality of this paper. We would especially like to thank them for the suggestions that gave rise to Section 4.2 and the discussion of intra-industry trade indicators in Section 2. Any remaining errors are of course the authors' responsibility. 


\section{References}

Bahmani-Oskooee, M., Harvey, H. (2006): How sensitive are Malaysia's Bilateral Trade Flows to Depreciation? Applied Economics, 38, 1279-1286

Balassa, B. (1965): Trade Liberalization and Revealed Comparative Advantage, The Manchester School, XXXIII, 99 - 123

Balassa, B. (1977): A Stages Approach to Comparative Advantage, Paper presented at the Fifth World Congress of the International Economic Association, Tokyo, 29 Aug. - 3 Sept., 1977

Balassa, B. (1987) Intra-industry Specialization: A Cross-Country Analysis, European Economic Review, 30, 27-42

Beran, B. and Ducharme, G.R. (1992): Asymptotic Theory for Bootstrap Methods in Statistics, Les Publication CRM, Centre de Recherches Mathématiques, Université de Montreal, Canada

Blind, K. and Jungmittag, A. (2005): Trade and the Impact of Innovation and Standards: The Case of Germany and the UK, Applied Economics, 37, 1385-1398

Brülhart, M. (1994): Marginal Intra-Industry Trade: Measurement and Relevance for the Pattern of Industrial Adjustment, Weltwirtschaftliches Archiv, 130, 600-613

De Benedictis, L. and Tamberi, M. (2001): A Note on the Balassa Index of Revealed Comparative Advantage, Working Paper SSRN 289602

De Benedictis, L., Gallegati, M. and Tamberi, M. (2008): Semiparametric Analysis of the Specialization-income Relationship, Applied Economic Letters, 15, 301-306 
Doroodian, K., Jung, C.H., and Boyd, R. (1999): The J-curve Effect and US Agricultural and Industrial Trade, Applied Economics, 31, 687-695

Engelsman, E.C. and van Raan, A.F.J. (1990): The Netherlands in Modern Technology: A Patent-based Assessment, Centre for Science and Technology Studies, University of Leiden

Garcia-Castrillo, P., Montanes, A. and Sanz-Gracia, F. (2002): A World-Wide Assessment of Economics (1992-1997), Applied Economics, 34, 1453-1475

Gray, P. (1979) Intra-industry Trade: The Effects of Different Levels of Data Aggregation, in On the Economics of Intra-industry Trade, (ed.) Giersch, H., Mohr, Tubingen

Grubel, H.G., Lloyd, P. (1971): The Empirical Measurement of Intra-industry Trade, Economic Record, 47, 494-517

Grubel, H.G., Lloyd, P. (1975): Intra-industry Trade: The Theory and Measurement of International Trade in Differentiated Products, John Wiley, New York

Grupp, H. (1998): Foundations of the Economics of Innovation, Elgar Cheltenham

Grupp, H. (1994): The measurement of technical performance of innovations by technometrics and its impact on established technology indicators, Research Policy, 23, $175-193$

Hall, P. (1992): The Bootstrap and Edgeworth Expansion, Springer, New York

Hayashi, F. (2000): Econometrics, Princeton University Press, New York

Horrowitz, J. (2001): The Bootstrap, in: J. J. Heckman and E. Learmer (eds.), Handbook of Econometrics, Vol. 5, Amsterdam

Jang, S.L., Tsai, Y.T. and Cheng, J. (2008): Persistent Innovation: A Cross-Country Study of Output and Diversity over Time, Applied Economics Letters, 15, 323-326

Jensen, C. (2002): Foreign Direct Investment, Industrial Restructuring and the Upgrading of Polish Exports, Applied Economics, 35, 207-217 
Keesing, D.B. (1965): Labor Skills and International Trade: Evaluating Many Trade Flows with a Single Measuring Device, Review of Economics and Statistics, 47, 287 - 294

Krugman, P.R. (1991): Geography and Trade, MIT Press

Serfling, R. (2002): Approximation Theorems of Mathematical Statistics, Wiley \& Sons

Tharakan, P.K.M. (1984) Intra-industry Trade between the Industrial Countries and the Developing World, European Economic Review, 26, 213-27

Wasserman, L. (2006): All of Nonparametric Statistics, Springer, New York

Wooldridge, J.M. (2002): Econometric Analysis of Cross Section and Panel Data, The MIT Press, Cambridge, Massachusetts

Wolter, F. (1977): Factor Proportions, Technology and West-German Industry's International Trade Patterns, Weltwirtschaftliches Archiv, 113, 250 - 267 


\section{Appendix}

\section{Proof of Theorem 1:}

Under conditions i) and ii) we have

$X_{i k} \sim B\left(1, \pi_{k}\right)$ with $E\left(X_{i k}\right)=\pi_{k}, V\left(X_{i k}\right)=\pi_{k}\left(1-\pi_{k}\right)$

as well as

$$
\sum_{i=1}^{n_{j}} X_{i j} \sim B\left(n_{j}, \pi_{j}\right) \text { with } E\left(\sum_{i=1}^{n_{j}} X_{i j}\right)=n_{j} \pi_{j}, V\left(\sum_{i=1}^{n_{j}} X_{i j}\right)=n_{j} \pi_{j}\left(1-\pi_{j}\right) \quad \forall j
$$

Check that the true value of the linear specialisation ratio can be defined by:

$$
r \equiv E\left(n_{k}^{-1} \sum_{i=1}^{n_{k}} X_{i k}\right) / E\left(n^{-1} \sum_{j=1}^{J} \sum_{i=1}^{n_{j}} X_{i j}\right)=\frac{\pi_{k}}{\sum_{j=1}^{J} \frac{n_{j}}{n} \pi_{j}} \equiv \frac{\pi_{k}}{\pi}
$$

This can be estimated by using the corresponding sample shares. To establish the asymptotics of the estimator $\hat{r}=\pi_{k} / \pi$, we first show that the estimated shares are normal.

We have:

$n_{j}^{-1} \sum_{i=1}^{n_{j}} X_{i j} \stackrel{d}{\rightarrow} N\left(\pi_{j}, \frac{\pi_{j}\left(1-\pi_{j}\right)}{n_{j}}\right)$

which follows from the central limit theorem. The linear indicator is thus a function of $J$ asymptotically normal variables. We use the mean value expansion (see Hayashi, 2000, Wooldridge, 2002) to derive the following statement:

$$
\hat{r}=r+\frac{\partial r}{\partial \vec{\pi}} \Delta \pi
$$


where $\partial r / \partial \vec{\pi}=\left(-\pi_{k} \frac{n_{1}}{n} / \pi^{2}, \ldots,\left(\pi-\frac{n_{k}}{n} \pi_{k}\right) / \pi^{2}, \ldots-\pi_{J} \frac{n_{J}}{n} / \pi^{2}\right)$ is the gradient evaluated at values for the shares caught between the true and the estimated. Also, note that $\Delta \pi=\left(\pi_{1}-\pi_{1}, \ldots, \pi_{J}-\pi_{J}\right)^{\prime}$. We obtain:

$$
\begin{aligned}
& \sqrt{n}(\hat{r}-r)=\frac{\partial r}{\partial \vec{\pi}} \sqrt{n} \Delta \pi+o_{p}(1) \\
& \stackrel{d}{\rightarrow} N\left(0, \frac{\partial r}{\partial \vec{\pi}} \operatorname{cov}(\sqrt{n} \pi) \frac{\partial r}{\partial \vec{\pi}}\right)
\end{aligned}
$$

From independence stated in ii) we find that the covariance matrix is diagonal:

$$
\operatorname{cov}(\sqrt{n} \pi) \equiv V=\operatorname{diag}\left(\frac{n}{n_{1}} \pi_{1}\left(1-\pi_{1}\right), \ldots, \frac{n}{n_{J}} \pi_{J}\left(1-\pi_{J}\right)\right)
$$

Thus we conclude that $\Delta \pi$ is $\sqrt{n}$-consistent when iii) holds. The variance can be calculated by straightforward matrix multiplication

$$
(\partial r / \partial \vec{\pi}) V(\partial r / \partial \vec{\pi})^{\prime}=n \sum_{j=1}^{J}\left(\frac{\partial r}{\partial \pi_{j}}\right)^{2} \frac{\pi_{j}\left(1-\pi_{j}\right)}{n_{j}}
$$

Now, again by the delta method under iv) any of the transformed versions is also normal. Let the transformation function be denoted by $I:{ }^{+} \rightarrow[a, b]$, where $[a, b]$ is some interval on , possibly even itself. Then we use the mean value theorem to get:

$$
\sqrt{n}(\hat{I}-I)=\left(\frac{d I}{d r} \frac{\partial r}{\partial \pi_{1}}, \ldots, \frac{d I}{d r} \frac{\partial r}{\partial \pi_{J}}\right) \sqrt{n} \Delta \pi+o_{p}(1) \stackrel{d}{\rightarrow} N\left(0, \sigma_{I}^{2}\right)
$$

with 


$$
\sigma_{I}^{2}=n \sum_{j=1}^{J}\left(\frac{d I}{d r} \frac{\partial r}{\partial \pi_{j}}\right)^{2} \frac{\pi_{j}\left(1-\pi_{j}\right)}{n_{j}}
$$

The important point with (9) and (10) is that we can treat $\hat{I}$ as approximately normal with $N\left(I, \sigma_{I}^{2} / n\right)$, where $\sigma_{I}^{2}$ is a consistent estimator of $\sigma_{I}^{2}$ usually obtained by replacing $\left(\pi_{k}, \pi\right)$ with the sample shares.

\section{Proof of Theorem 2:}

Define $\Delta I \equiv I_{v}-I_{k}=I_{v}\left(r_{v}\left(\pi_{v}, \pi\right)\right)-I_{k}\left(r_{k}\left(\pi_{k}, \pi\right)\right)$. It is easy to verify that:

$$
\sqrt{n}(\Delta I-\Delta I)=\frac{\partial \Delta I}{\partial \vec{\pi}} \sqrt{n} \Delta \pi+o_{p}(1) \stackrel{d}{\rightarrow} N\left(0, \frac{\partial \Delta I}{\partial \vec{\pi}} V \frac{\partial \Delta I^{\prime}}{\partial \vec{\pi}}\right)
$$

where we obtain the gradient of $\Delta I$ by:

$$
\begin{aligned}
\frac{\partial \Delta}{\partial \vec{\pi}}=\left(-\frac{d I_{v}}{d r_{v}} \frac{\pi_{v} \frac{n_{1}}{n}}{\pi^{2}}+\frac{d I_{k}}{d r_{k}} \frac{\pi_{k} \frac{n_{1}}{n}}{\pi^{2}}, \ldots, \frac{d I_{v}}{d r_{v}} \frac{\pi-\pi_{v} \frac{n_{v}}{n}}{\pi^{2}}+\frac{d I_{k}}{d r_{k}} \frac{\pi_{k} \frac{n_{v}}{n}}{\pi^{2}}, \ldots,\right. \\
\left.-\frac{d I_{v}}{d r_{v}} \frac{\pi_{v} \frac{n_{k}}{n}}{\pi^{2}}-\frac{d I_{k}}{d r_{k}} \frac{\pi-\pi_{k} \frac{n_{k}}{n}}{\pi^{2}}, \ldots,-\frac{d I_{v}}{d r_{v}} \frac{\pi_{v} \frac{n_{J}}{n}}{\pi^{2}}+\frac{d I_{k}}{d r_{k}} \frac{\pi_{k} \frac{n_{J}}{n}}{\pi^{2}}\right)
\end{aligned}
$$

as well as $V$ is given by

$$
(\partial \Delta I / \partial \vec{\pi}) V(\partial \Delta I / \partial \vec{\pi})^{\prime}=n \sum_{j=1}^{J}\left(\frac{\partial \Delta I}{\partial \pi_{j}}\right)^{2} \frac{\pi_{j}\left(1-\pi_{j}\right)}{n_{j}}
$$




\section{Proof of Theorem 3:}

To compare indicators over time is conceptually very much the same. The proof follows along the lines of that of Theorem 2. We therefore omit most of it. However, since $\Delta I$ consists of twice as many different estimators for the sample shares, the variance is slightly different:

$$
\sigma_{I}^{2} \equiv n^{t} \sum_{j=1}^{J}\left[\left(\frac{\partial I^{s}}{\partial \pi_{j}^{s}}\right)^{2} \frac{\pi_{j}^{s}\left(1-\pi_{j}^{s}\right)}{n_{j}^{s}}+\left(\frac{\partial I^{t}}{\partial \pi_{j}^{t}}\right)^{2} \frac{\pi_{j}^{t}\left(1-\pi_{j}^{t}\right)}{n_{j}^{t}}\right]
$$

where the derivatives of the transformations are collected in the following vector:

$$
\frac{\partial \Delta I}{\partial \vec{\pi}}=\left(-\frac{d I^{t}}{d r^{t}} \frac{\pi_{k}^{t} \frac{n_{1}^{t}}{n^{t}}}{\pi^{t 2}}, \ldots, \frac{d I^{t}}{d r^{t}} \frac{\pi^{t}-\pi_{k}^{t} \frac{n_{k}^{t}}{n^{t}}}{\pi^{t 2}}, \ldots,-\frac{d I^{t}}{d r^{t}} \frac{\pi_{k}^{t} \frac{n_{J}^{t}}{n^{t}}}{\pi^{t 2}}, \frac{d I^{s}}{d r^{s}} \frac{\pi_{k}^{s} \frac{n_{1}^{s}}{n^{s}}}{\pi^{s 2}}, \ldots,-\frac{d I^{s}}{d r^{s}} \frac{\pi^{s}-\pi_{k}^{s} \frac{s_{k}^{s}}{n^{s}}}{\pi^{s 2}}, \ldots, \frac{d I^{s}}{d r^{s}} \frac{\pi_{k}^{s} \frac{n_{J}^{s}}{n^{s}}}{\pi^{s 2}}\right)
$$

\section{Proof of Theorem 4:}

We will not discuss the testing problem explicitly because a confidence interval that entails the value of the null hypothesis is indicative of a non-ejection decision with the corresponding test. First, we will define the bootstrap algorithm and then argue why it is consistent.

- Given $X_{j}=\left(X_{j 1}, \ldots, X_{j n_{j}}\right)$ for all $j$, calculate $\theta_{n}$, where $\theta_{n}$ is one of the estimators described in Theorem 1-3.

- Draw from each vector $X_{j} n_{j}$ elements randomly with replacement. From this form the bootstrap sample $X_{j}^{*}=\left(X_{j 1}^{*}, \ldots, X_{j n_{j}}^{*}\right)$.

- Calculate $\left|T_{n}^{*}\right|=\left|\sqrt{n}\left(\theta_{n}^{*}-\theta_{n}\right) / s_{n}^{*}\right| \quad$ for the two-sided alternative or 
$T_{n}^{*}=\sqrt{n}\left(\theta_{n}^{*}-\theta_{n}\right) / s_{n}^{*}$, where $\theta_{n}^{*}$ and $s_{n}^{*}$ are the bootstrap point estimator and its corresponding standard deviation. The latter will be calculated by one of equations (10), (13) or (14). 16

- Repeat the last two steps very often.17 Take from the resulting bootstrap EDF the $1-\alpha$-quantile, $z_{n, \alpha / 2}^{*}$.

- Define the bootstrap confidence interval as $\theta_{n} \pm z_{n, \alpha / 2}^{*} s_{n} / \sqrt{n}$, where $s_{n}$ can be calculated by one of (10), (13) or (14) based on the original sample.

It should be noted that this approach follows a non-parametric re-sampling strategy, which might seem curious at first sight, because we have specified a parametric distribution. However, since the binomial distribution has only two outcomes (success and failure), both techniques have identical statistical properties, which is not the case with any other common statistical distribution.

Concerning consistency, it is shown by Beran and Ducharme (1992) that we need to meet three conditions:

- $\sup _{x}\|F(x)-F(x)\|^{p} \rightarrow 0$, i.e. that the EDF converges uniformly to the CDF.

- $G_{\infty}(x, F)$, denoting the asymptotic CDF of the statistic, is continuous.

16 In fact, we could use a nested bootstrap to determine the variance. But this is computationally even more intensive. Since we are in the lucky situation of having an asymptotic expression for the variance it is better to use this for practical reasons (see e.g. Wassermann (2006)).

17 The precision increases with the number of bootstrap replications. Thus infinite replications would be ideal. Since this is impossible for reasons of finite computation time, a usual recommendation is to choose $1,000-5,000$ replications. 
- for each sequence $\left\{H_{n}\right\}$ out of properly defined neighbourhood of $F$, such that $H_{n}$ converges to $F, \lim _{n \rightarrow \infty} G_{n}\left(x, H_{n}\right)=G_{\infty}(x, F)$.

Checking the second and third condition is absolutely standard. The second holds because we know that the distribution of the statistics under consideration are asymptotically $N(0,1)$. Therefore, $G_{\infty}(x, F)=N(0,1)$ in the one-sided interval and when we use the two-sided interval, $G_{\infty}(x, F)=H N(\sqrt{2 / \pi}, \sqrt{(\pi-2) / \pi}) 18$. Turning to the third condition, we use Polya's Theorem which states that for any $X_{n}$ converging to $X$ almost surely, if the corresponding distribution function $Z_{X}$ is continuous, then $\limsup _{n \rightarrow \infty} \sup _{x}\left(\left|Z_{X_{n}}(x)-Z_{X}(x)\right|\right)=0$. Along with condition 2, we must have $\limsup _{n \rightarrow \infty} \sup _{x}\left(\left|G_{n}\left(x, H_{n}\right)-G_{\infty}(x, F)\right|\right)=0$, which implies the last condition. Combining this with the first condition (if it holds), it follows that $\lim _{n \rightarrow \infty} P\left(\sup _{x}\left(\left|G_{n}(x, F)-G_{\infty}(x, F)\right|\right)>\varepsilon\right)=0 . \quad$ The first $\quad$ condition requires $\sup _{x}\|F(x)-F(x)\| \stackrel{p}{\rightarrow} 0$, where $F(\cdot)$ is vector of $J$ distribution functions corresponding to the distribution of the objects in each of the $j$ subjects. Because of the Glivenko-Cantelli Theorem we have component-wise convergence. However, with the Euclidean distance this is enough (see also Serfling, 2002). Check that:

$18 \pi$ now denotes the circle constant. 


$$
\begin{aligned}
P\left(\sup _{x} \|\right. & F(x)-F(x) \|<\varepsilon)=P\left(\sup _{x}\left(\sum_{j=1}^{J}\left(F_{j}(x)-F_{j}(x)\right)^{2}\right)^{1 / 2}<\varepsilon\right) \\
& \geq P\left(\sup _{x} \sum_{j=1}^{J}\left|F_{j}(x)-F_{j}(x)\right|<\varepsilon\right) \geq P\left(\sum_{j=1}^{J} \sup _{x_{j}}\left|F_{j}\left(x_{j}\right)-F_{j}\left(x_{j}\right)\right|<\varepsilon\right) \rightarrow 1
\end{aligned}
$$

Thus all conditions are met and the bootstrap is consistent. 